\title{
Dynamic VAR planning methodology to enhance transient voltage stability for failure recovery
}

\author{
Di YANG ${ }^{1}$, Haozhong CHENG $^{2}$, Zeliang $\mathrm{MA}^{3}$, Liangzhong YAO ${ }^{4}$, \\ Zhonglie $\mathbf{Z H U}^{3}$
}

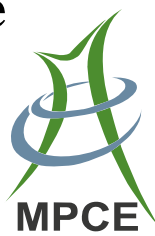

\begin{abstract}
In recent years, failure recovery after faults is concerned and discussed worldwide as an important and hot topic. Facing the challenge of heavy loads and ultrahigh voltage transmission, it's urgent to propose some solutions to enhance transient voltage stability for failure recovery. Therefore, a novel dynamic volt ampere reactive (VAR) planning methodology is proposed in this paper to help failure recovery and improve transient voltage stability after contingencies. First, a transient voltage fluctuation (TVF) index is proposed to evaluate transient voltage condition after faults. Then dynamic compensation
\end{abstract}

CrossCheck date: 30 July 2017

Received: 30 April 2016/ Accepted: 30 July 2017/Published online: 28 December 2017

(C) The Author(s) 2017. This article is an open access publication

$\triangle$ Di YANG

yangti1983@gmail.com

Haozhong CHENG

hzcheng@sjtu.edu.cn

Zeliang MA

zlma@ec.sgcc.com.cn

Liangzhong YAO

yaoliangzhong@epri.sgcc.com.cn

Zhonglie ZHU

zhuzl@ec.sgcc.com.cn

1 State Grid Pudong Power Supply Company, SMEPC, Pudong District, Shanghai 200122, China

2 Department of Electronic Information and Electrical Engineering, Shanghai Jiao Tong University, Minhang District, Shanghai 200240, China

3 East China Grid Company Limited, Pudong District, Shanghai 200120, China

4 China Electric Power Research Institute, Haidian District, Beijing 100192, China sensitivity is presented for searching the best candidate locations. Following that, the dynamic VAR planning methodology based on an improved Tent chaos multi-objective algorithm is discussed in detail. There are two optimization objects in the optimization. One is to minimize TVF to enhance transient voltage stability for failure recovery. And the other optimization is to minimize dynamic VAR investment cost and operation cost. Finally, IEEE 39 power system and a practical power system are analyzed and discussed. The proposed dynamic VAR planning methodology can support enough reactive power for failure recovery. With the least SVC planning amount and the power loss cost, it can greatly decrease the system TVF index and enhance the transient voltage stability. It's proved that the proposed dynamic VAR planning optimization is effective and helpful for safety operation of power system.

Keywords Dynamic VAR planning, Failure recovery, Improved Tent mapping chaos optimization, Transient stability

\section{Introduction}

Nowadays, transient voltage stability is concerned and investigated more and more worldwide due to the challenges from the development of network topology and extra-high voltage transmission. Previous researches mostly focus on static voltage stability [1-3] rather than transient voltage stability. In order to improve transient voltage stability and help failure recovery, static volt ampere reactive (VAR) compensator (SVC) and static synchronous compensator (STATCOM) are some good devices to avoid voltage instability risk $[4,5]$. 
For evaluating transient voltage stability, some assessment indexes such as voltage dip index [6] and transient voltage severity index [7] have been proposed. They can be used in transient voltage stability analysis and dynamic VAR optimization. Dynamic VAR planning optimization is an approach to enhance transient voltage stability. However, some transient voltage stability indexes are not suitable for dynamic VAR planning. Therefore, a good and suitable transient voltage stability can be well used in the dynamic VAR planning optimization and enhance failure recovery for power system after faults.

There are two main issues in dynamic VAR planning optimization. One is to choose the best dynamic VAR installation candidate locations. The other issue is to build a good optimization model and methodology to enhance transient voltage stability for failure recovery. Trajectory sensitivity is commonly used for dynamic VAR installation locations [8-11]. And candidate locations for dynamic VAR can also be determined through reactive power optimization.

In the dynamic VAR optimization researches, optimal power flow (OPF) considering rotor stability is developed and investigated [11-16]. In [17], the OPF problem considers voltage stability as a constraint. But transient voltage stability has not been considered in the literature. In [11], a long term dynamic VAR allocation and dynamic optimization method are analyzed and discussed in detail. Most of the dynamic VAR optimization methodologies formulate the transient voltage stability model as a constraint rather than an optimization objective.

There have been many reactive power planning methodologies proposed in literatures. However, most of the researches focused on the static voltage stability improvement. Through some of the dynamic VAR optimization methodologies have been proposed, the transient voltage assessment indexes used in these methodologies such as transient voltage dip only considered some transient characteristics. Some researches have used some sensitivities (such as trajectory sensitivity) to evaluate the transient voltage influence. But the optimization is a static optimization in essence. The dynamic VAR planning methodology proposed in this paper considers the transient voltage recovery process. The optimization considers transient effect in every optimization iteration. And the methodology can not only enhance the transient voltage but also accelerate the recovery after faults.

Dynamic VAR planning methodology to enhance transient voltage stability is a good choice for failure recovery after faults. The contribution of this paper is as following:

1) TVF index is presented to reflect the conditions of failure recovery after contingencies. And a compensation sensitivity approach is presented for dynamic VAR candidate locations.

2) A dynamic VAR planning optimization methodology based on an improved Tent mapping chaos algorithm is proposed and analyzed for failure recovery after faults in this paper.

\section{Transient voltage stability assessment}

Transient voltage stability assessment indexes are useful to evaluate the voltage stability condition under contingencies. Some assessments such as maximum fault clearing time and the lowest voltage during voltage recovery are usually adopted to evaluate transient voltage stability. Besides these indexes, a novel index, TVF assessment index, is proposed in this paper. TVF index can reflect the failure recovery condition after contingencies.

Transient voltage after a large disturbance is shown in Fig. 1. Voltage will fluctuate seriously after a large disturbance if voltage is unstable. And this situation means that failure recovery cannot achieve well after faults. Otherwise, voltage will fluctuate little and tend to stable rapidly. Therefore, the voltage fluctuation condition can be observed to evaluate voltage stability condition. Then TVF index is presented as (1):

$T V F_{i}^{F_{k}}=\frac{1}{2}\left(\frac{1}{N_{T}} \sum_{p=1}^{N_{T}} V_{i, \max }^{F_{k}, N_{p}}-\frac{1}{N_{T}} \sum_{p=1}^{N_{T}} V_{i, \text { min }}^{F_{k}, N_{p}}\right)$

where $N_{T}$ is the cycle number of transient simulation; $N_{p}$ is the $p$ th simulation cycle after fault; $V_{i, \max }^{F_{k}, N_{p}}, V_{i, \min }^{F_{k}, N_{p}}$ are the maximum and minimum voltage of bus $i$ at the $N_{p}$ cycle after the $F_{k}$ fault; $F_{k}$ is the $k$ th fault in faults set $\Re_{F}$.

TVF is an average transient voltage recovery fluctuation assessment index. In order to evaluate transient voltage

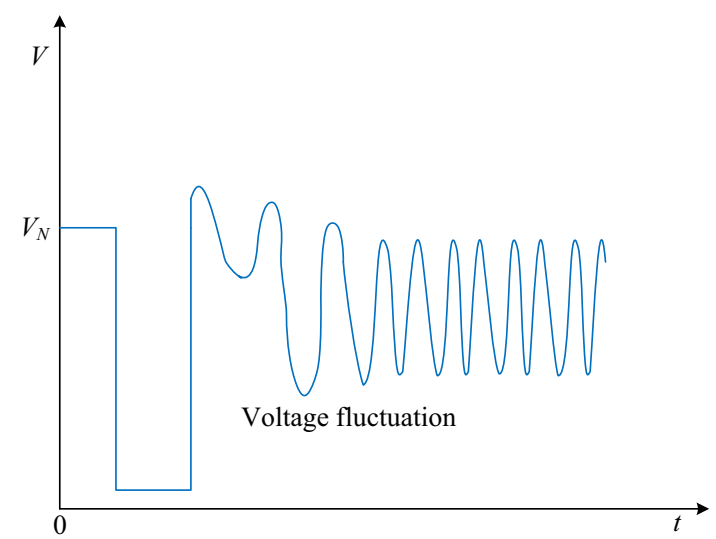

Fig. 1 Transient voltage after a large disturbance 
conditions of all the power system, system TVF is presented as (2):

$T V F_{s}^{F_{k}}=\max \left(T V F_{i}^{F_{k}}\right)$

The proposed index can show the fluctuation after faults. And the index is used to optimize the system transient stability under serious faults condition. The serious faults is the faults that will cause voltage instability. In this situation, the transient voltage will fluctuate greatly. So the condition for the utilization of the proposed index is to pick out the serious faults and areas first.

TVF can be used in the transient voltage stability optimization problem. The approach to choose the best candidate locations of dynamic VAR is a key for the optimization problem. SVC as an important dynamic VAR compensator is analyzed in this paper. The compensation sensitivity method is presented as (3) for SVC candidate locations.

$S_{T V F_{s}}^{F_{k}}=\frac{\partial T V F_{s}^{F_{k}}}{\partial Q_{s v c}}$

In order to simplify the SVC candidate approach, the sensitivity can be transformed as (4):

$S_{T V F_{s}}^{F_{k}}=\frac{\Delta T V F_{s}^{F_{k}}}{\Delta Q_{s v c}}$

Then the candidate approach for dynamic VAR can be solved and searched conveniently.

\section{Formulation of dynamic VAR planning problem}

In order to enhance transient voltage stability for failure recovery under contingencies, a dynamic VAR planning methodology is proposed in this paper. The objective function and constraint condition are discussed respectively in this section.

\subsection{Objective function}

The optimization goal is to enhance the transient voltage stability for failure recovery with the most economic investment and operation cost.

The optimization is formed based on two objective functions. One of the objective functions is to minimize the TVF index under a contingency, which is presented as (5):

$\min f_{1}(x)=T V F_{s}$

The system TVFs can be solved based on (1). Another optimization objective function presented as (6) is to minimize SVC investment cost and power loss cost. $\min f_{2}(x)=C_{e} \zeta P_{l o s s}+\frac{1}{T_{l}} \sum_{i \in \Re_{s v c}}\left(C_{s v c, i} Q_{s v c, i}+F_{s v c, i}\right)$

where $C_{e}$ is the electric price; $\zeta$ is the operation hours at the year maximum load; $P_{\text {loss }}$ is the power loss; $T_{l}$ is the lifetime of SVC; $Q_{s v c, i}$ is the compensation capacity of SVC at bus $i$; $C_{s v c, i}$ is the investment cost of SVC; $F_{s v c, i}$ is the installation and building cost of SVC; $\Re_{s v c}$ is the buses of SVC that are the candidate locations.

As the optimization objective in the paper, one of the objective functions is to minimize the TVFs index under a contingency, which is presented as (5). Another optimization objective function presented as (6) is to minimize SVC investment cost and power loss cost. When the system is unstable, the TVFs is much bigger than the stable condition. The objective function as (5) can make the TVFs tend smaller. It means that the optimization result can make the fluctuation smaller and the system easier to recovery.

TVFs as one of the optimization are used to decrease the fluctuation. The unstable solutions in the iteration will be gradually eliminated. Thus the optimization result can greatly improve the stability.

\subsection{Constraint conditions}

The constraint conditions are consisted of dynamic response constraint, power equilibrium constraint, rotor angle constraint and inequality constraint.

1) Dynamic response constraint

The dynamic responses such as generators and SVC can be presented as (7):

$\left\{\begin{array}{l}\dot{x}=f(x, y, u) \\ g(x, y, u)=0\end{array}\right.$

where $x, y, u$ are the state variable, algebraic variable and control variable respectively; $f(x, y, u)$ is the model to describe the dynamic responses of dynamic elements; $g(x, y, u)$ is the model of network topology.

Of course, the initial operation needs satisfying as (8):

$g\left(x_{0}, y_{0}, u_{0}\right)=0$

where $x_{0}, y_{0}, u_{0}$ are the initial state of the variables.

The active power and reactive power of generator are presented as (9)-(10):

$P_{i, t}^{G}=\frac{E_{i} V_{i, t}}{X_{d, i}^{\prime}} \sin \left(\delta_{i, t}-\theta_{i, t}\right)$

$Q_{i, t}^{G}=\frac{E_{i} V_{i, t}}{X_{d, i}^{\prime}} \cos \left(\delta_{i, t}-\theta_{i, t}\right)-\frac{\left(V_{i, t}\right)^{2}}{X_{d, i}^{\prime}}$

where $E_{i}$ is the electric potential of generators; $X_{d, i}^{\prime}$ is the transient reactance in direct axis; $V_{i, t}, \delta_{i, t}, \theta_{i, t}$ are voltage, 
rotor angle and voltage angle of generator $i$ at $t$ time respectively.

The reactive power of SVC is presented as (11):

$Q_{i, t}^{s v c}=\left(\omega C-\frac{2 \beta_{t}-\sin 2 \beta_{t}}{\pi \omega L}\right) V_{i, t}^{2}$

where $Q_{i, t}^{s v c}$ is the reactive power of the $i$ th SVC at $t$ time; $\omega$ is the angular frequency; $C$ and $L$ are capacitor and reactor of SVC; $\beta_{t}$ is the trigger angle of SVC.

2) Power equilibrium constraint

Power equilibrium of power system needs satisfying (12)-(13):

$$
P_{G, i}-P_{L, i}=V_{i} \sum_{j=1}^{N_{b}}\left(G_{i j} \cos \left(\theta_{i}-\theta_{j}\right)+B_{i j} \sin \left(\theta_{i}-\theta_{j}\right)\right) V_{j}
$$

$$
\begin{aligned}
Q_{G, i}+Q_{s v c, i}-Q_{L, i}= & V_{i} \sum_{j=1}^{N_{b}}\left(G_{i j} \sin \left(\theta_{i}-\theta_{j}\right)\right. \\
& \left.-B_{i j} \cos \left(\theta_{i}-\theta_{j}\right)\right) V_{j}
\end{aligned}
$$

where $P_{G, i}, P_{L, i}, Q_{G, i}, Q_{L, i}$ are the active power and reactive power of generators and loads, respectively; $G_{i j}, B_{i j}$ are the conductance and reactance of branches; $Q_{s v c, i}$ is the reactive power compensation amount of SVC; $V_{i}, V_{j}, \theta_{i}, \theta_{j}$ are voltage amplitude and voltage angle of buses $i$ and $j$ respectively; $N_{b}$ is the total number of buses.

\section{3) Rotor angle constraint}

Rotor angle of generators also needs satisfying as (14) in the dynamic VAR planning optimization for failure recovery besides enhancing transient voltage stability.

$\max \left(\Delta \delta^{t}\right) \leq \mu$

where $\Delta \delta^{t}$ is the rotor angle difference between generators; $\mu$ is the rotor angle difference threshold; $\mu$ is chosen to be $\pi$ in this paper.

\section{4) Inequality constraint}

Reactive power of generators and SVC needs satisfying as (15) and (16):

$Q_{G, i, \min } \leq Q_{G, i} \leq Q_{G, i, \max }$

$Q_{s v c, i \text { min }} \leq Q_{s v c, i} \leq Q_{s v c, i, \max }$

where $Q_{G, i, \min }, Q_{G, i, \max }$ are the minimum and maximum reactive power of generator respectively; and $Q_{s v c, i, \min }$, $Q_{s v c, i, \max }$ are the minimum and maximum reactive power of SVC respectively.

Bus voltage needs satisfying as (17):

$V_{i, \text { min }} \leq V_{i} \leq V_{i, \text { max }}$ where $V_{i, \min }, V_{i, \max }$ are the minimum and maximum voltage.

\section{Multi-objective optimal algorithm}

There have been lots of algorithms contributed to the optimization problems [18-23]. However, the optimization efficiency and the quality of the solutions need improving further. Therefore, an improved Tent mapping chaos optimization (ITMCO) is took part in the multi-objective optimization algorithm in this paper. It can solve the optimization problems with slow convergence rate and easy earliness. And the optimization algorithm can overcome the defect that some algorithms are not broad enough.

\subsection{Non-dominated sorting mechanism}

The key of multi-objective optimization is to search a global Pareto optimal solution set. The Pareto dominated solutions $X_{1}$ and $X_{2}$ is defined as $X_{1} \prec X_{2}$ when it is satisfied as (18):

$\begin{cases}f_{i}\left(X_{1}\right) \leq f_{i}\left(X_{2}\right) & \forall i \in[1, n] \\ f_{i}\left(X_{1}\right)<f_{i}\left(X_{2}\right) & \exists i \in[1, n]\end{cases}$

where $f_{i}(\cdot)$ is the $i$ th objective function; and $n$ is the number of objective functions.

Then Pareto optimal solutions set can be presented as:

$P_{s}=\left\{X \mid X_{i} \prec X\right\}$

Individuals in the population can be ranked based on non-dominated sorting mechanism. First, non-dominated individuals are sorted and ranked. Then the non-dominated degree of individuals at front ranking groups is stronger more than other individuals.

In addition, elite reservation strategy can be adopted based on crowding distance between individuals. Crowding distance is given as (20)-(21):

$\xi_{i}=\sum_{j=1}^{n} \xi_{i, j}$

$\xi_{i, j}=\frac{f_{i+1, j}-f_{i-1, j}}{f_{j, \max }-f_{j, \min }}$

where $\xi_{i}$ is crowding distance of individual $i$; $\xi_{i, j}$ is crowding distance of individual $i$ at the $j$ th objective function; $f_{i+1, j}, f_{i-1, j}$ are the $j$ th objective function of the previous and latter individuals; and $f_{j, \max }, f_{j, \min }$ are the maximum and minimum of the $j$ th objective function.

After sorted descending through crowding distance for neighbor individuals of all non-dominated individuals, the 
$N_{p}$ individuals at the front are chosen to form an offspring population.

\subsection{Improved Tent mapping chaos optimization}

In order to overcome the defect that non-dominated sorting algorithm is not broad enough, an improved Tent mapping chaos optimization (ITMCO) is took part in the multi-objective optimization algorithm [24].

Tent mapping is a one-dimensional piecewise linear mapping. And it can be presented as (22):

$X_{k+1}=T\left(X_{k}\right)= \begin{cases}2 X_{k} & 0 \leq X_{k} \leq 0.5 \\ 2\left(1-X_{k}\right) & 0.5<X_{k} \leq 1\end{cases}$

Tent mapping is simple and ergodic with uniformity. But some unstable cycle points still exist. Therefore, Tent mapping is improved as (23) if $X_{k}$ is $0,0.25,0.5,0.75$ or $X_{k}=X_{k-m}$.

$X_{k+1}=T^{\prime}\left(X_{k}\right)=T\left(X_{k}\right)+0.1 \cdot \operatorname{rand}(0,1)$

where $\operatorname{rand}(0,1)$ is a random function in range 0 and 1 . The distribution function of improved Tent mapping is symmetrical and effective. The chaos variables based on improved Tent mapping are mapped to the value space of optimization variables.

\subsection{ITMCO optimization algorithm flow}

Based on non-dominated sorting and ranking mechanism, elite reservation strategy, and improved chaos map optimization, the ITMCO algorithm is presented as:

Step 1: Set the population scale $N_{p}$, the maximum iteration number $K_{\max }$ and initialize population $P_{G}$.

Step 2: Generate chaos vectors $\alpha_{i, j}$ as (22)-(23) based on improved Tent mapping chaos multi-objective optimization. Then chaos mapping variables can be obtained through (24):

$X_{i, j}=X_{j, \min }+\alpha_{i, j}\left(X_{j, \max }-X_{j, \min }\right)$

where $X_{j, \max }, X_{j \text {, min }}$ are the maximum and minimum values of variables.

Step 3: Generate offspring population $Q_{G}$ with scale $N_{p}$ after selection, crossover and mutation operation.

Step 4: Merge parent population $P_{G}$ and offspring population $Q_{G}$ into a new population $R_{G}$. Perform nondominated sorting operation and crowding distance operator for $R_{G}$ to obtain the Pareto optimal solutions.

Step 5: Determine if the individuals' number at rank 1 is equal to the population scale $N_{p}$. If yes, the new population is the most optimal solution. Otherwise, choose $10 \%$ individuals $\widehat{X}_{i, j}$ of offspring population to search further adaptively. And $X_{i, j}$ can be obtained in a narrow interval as (25):

$\left\{\begin{array}{l}X_{j, \text { min }}^{\prime}=\widehat{X}_{i, j}-v\left(X_{j, \text { max }}-X_{j, \text { min }}\right) \\ X_{j, \text { max }}^{\prime}=\widehat{X}_{i, j}+v\left(X_{j, \text { max }}-X_{j, \text { min }}\right)\end{array}\right.$

where $v$ is the shrink factor and can be set in range 0 and 0.05 .

A new chaos vector $\beta_{i, j}$ can be generated based on (22)(23) to map a new decision variable. Then a new adaptive decision variable is presented as (26):

$X_{i, j}^{\prime \prime}=\omega X_{i, j}+(1-\omega) X_{i, j}^{\prime}$

where $\omega$ is the adaptive factor. It is presented as (27):

$\omega=1-\left(\frac{K-1}{K}\right)^{\tau}$

where $K$ is the iteration number. $\tau$ can be chosen through the objective functions, here $\tau$ is chosen to be 2 .

Step 6: Execute and search the optimization solutions based on Step 2-5 until the optimal solutions are obtained or the iteration number reaches the maximum number.

\subsection{Dynamic VAR planning optimization flow}

The dynamic VAR planning optimization is solved based on ITMCO algorithm. The optimization flow shown in Fig. 2 is discussed as follows:

Step 1: Input the topology structure data of power system and transient parameters data (generators, excitation system, and SVC). Set the maximum iteration number and the population parameters for ITMCO algorithm. Set the initial iteration number to be zero.

Step 2: Solve SVC investment cost and power loss cost at normal condition under the present individuals. And compute TVFs index after faults based on transient time domain simulation program. Then evaluate the optimal solution for the transient voltage stability, power loss cost and SVC investment cost objective functions in the evolution process.

Step 3: Search the more optimal solutions based on ITMCO algorithm. Generate chaos vectors $\alpha_{i, j}$ based on (22)-(23) and solve chaos mapping variables based on (24). Then perform the selection, crossover and mutation operation for individuals. And a new adaptive decision variable can be generated based on (26)-(27).

Step 4: Update individuals and iteration number. If the iteration number is smaller than $K_{\max }$, jump step-2 to solve the objective functions and continue to search the optimal solutions gradually. Otherwise, output the optimal compensation scheme. 


\section{Example analysis}

IEEE 39 power system and a practical power system in China are discussed and analyzed respectively to improve the transient voltage and failure recovery after faults.

In this paper, the parts are separated from the serious faults, which will make the system unstable. The serious faults can be picked out through transient time domain simulation. Then the areas are separated through the distribution of the serious faults set. The neighboring serious faults are put together as an area. The system in case study in the paper is separated into several parts so that the optimization problem can be decomposed and the best optimization solutions can be searched faster and more easily.

\subsection{IEEE 39 power system}

Transient voltage stability problem for failure recovery is an important issue on the dynamic VAR planning. IEEE

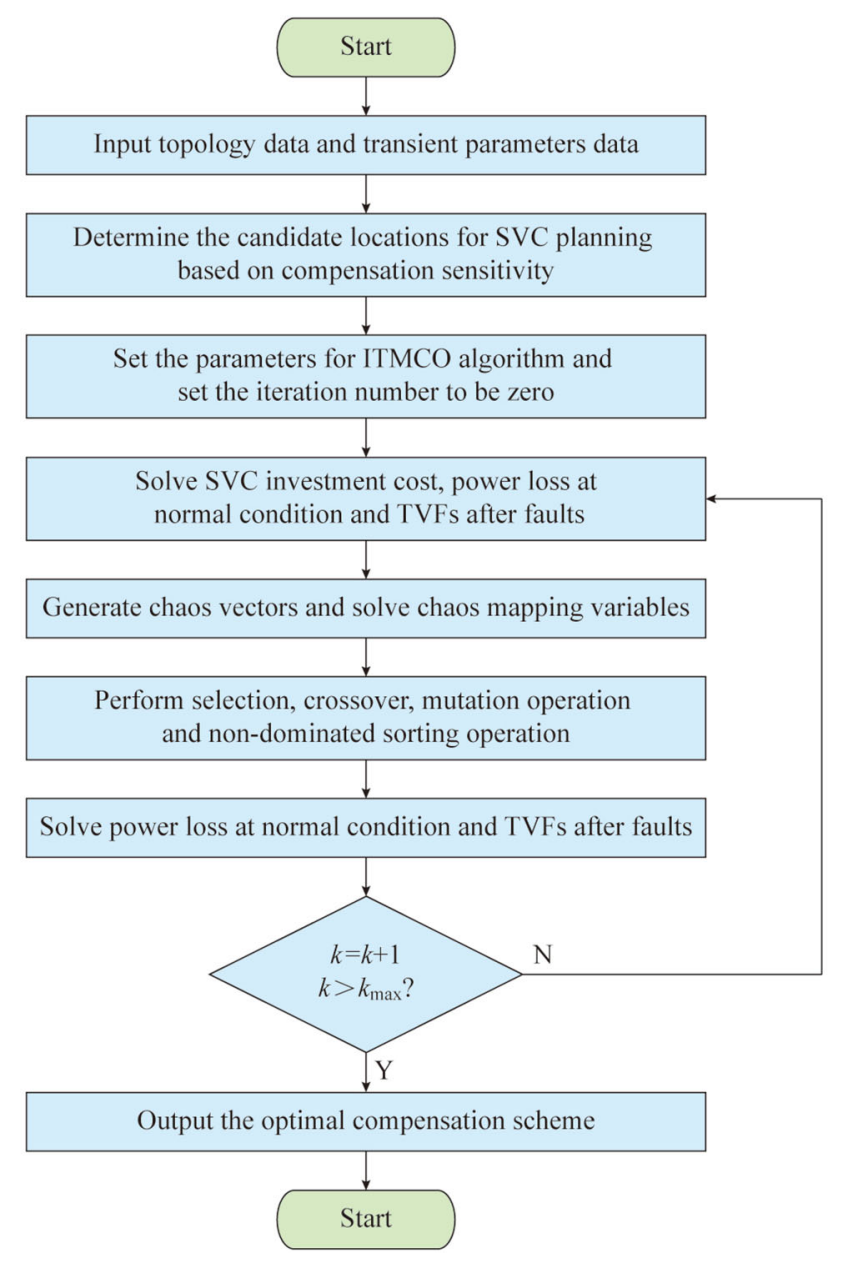

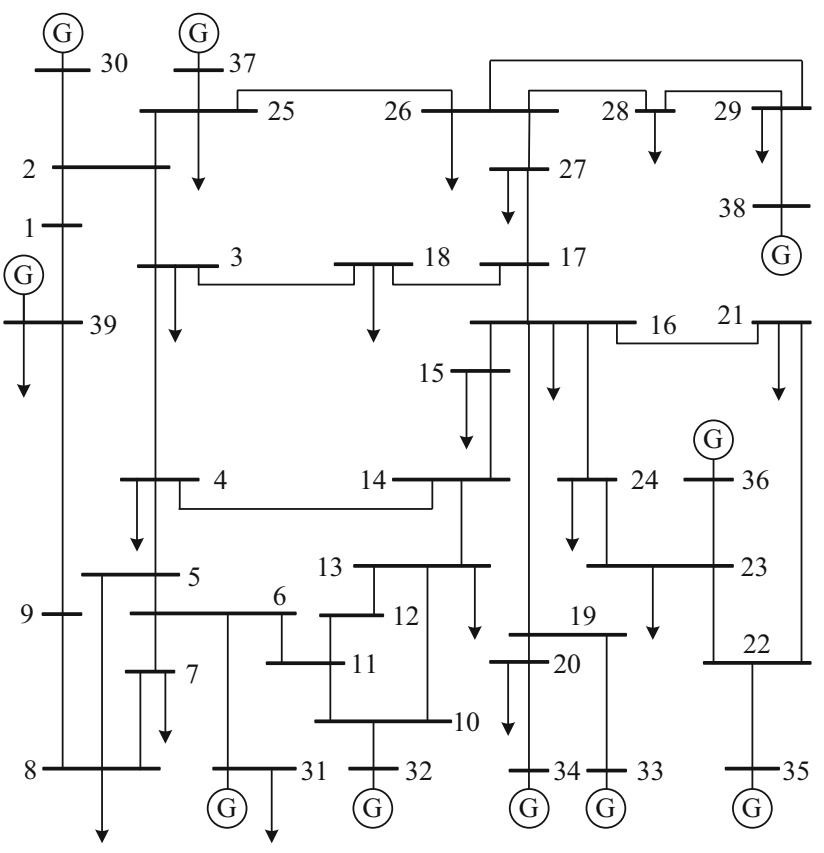

Fig. 3 IEEE 39 power system

39 power system shown in Fig. 3 is illustrated and analyzed in this paper.

Based on transient time domain simulation, the weak branches that will bring voltage instability problems are located at area 1 (branch 10-11, 10-13) and area 2 (branch 25-26, 25-37, 26-27, 26-28, 26-29, 28-29).

Before the dynamic VAR planning optimization, good dynamic VAR candidate locations need determining based on the proposed compensation sensitivity as (4). The compensation sensitivities for weak areas under different

Table 1 Compensation sensitivity for weak areas under different installation locations

\begin{tabular}{clll}
\hline Area & Compensation bus1 & Compensation bus2 & $S_{T V F_{s}}$ \\
\hline 1 & 10 & - & 0.0113 \\
& 11 & - & 0.0114 \\
& 13 & - & 0.0552 \\
& 6 & - & 0.0104 \\
& 4 & - & 0.0549 \\
2 & 26 & 28 & 0.0073 \\
& 26 & 29 & 0.0102 \\
& 28 & 29 & 0.0080 \\
& 26 & 27 & 0.0619 \\
& 27 & 28 & 0.0616 \\
& 26 & 25 & 0.0632 \\
& 25 & - & 0.0576 \\
& 26 & - & 0.0630 \\
& 27 & - & 0.0552 \\
\hline
\end{tabular}

Fig. 2 Optimization flow for dynamic VAR planning 
installation locations are presented in Table 1. It's obvious that buses 11, 28 and 29 are the best candidate locations for dynamic VAR planning.

The optimization objective is to minimize index TVFs for enhancing transient voltage stability with the least SVC investment and power loss cost and helping failure recovery. The parameters for dynamic VAR planning optimization are shown in Table 2. There are two optimization objectives. One is to minimize TVF as (5). And another objective is to minimize the investment and power loss cost as (6). The planning optimization is solved based on improved Tent mapping chaos multi-objective optimization algorithm. The population scale $N_{p}$ is 45 . The maximum iteration number $I_{\max }$ is 300 . SVC compensation amount $Q_{s v c}$ is set in range 0-2000 Mvar. The limit of bus voltage is in range 0.9-1.1.

Before dynamic VAR planning optimization, there are some branch faults with voltage instability problems. For example, transient voltage under branch $26-27$ fault is shown in Fig. 4. The fault time is $100 \mathrm{~ms}$. After the dynamic VAR planning optimization, transient voltage can recover and achieve a new equilibrium condition under each of branch faults. Take branch 26-27 fault for an example, transient voltage of all buses is stable with SVC optimization amount, which is shown in Fig. 5.

The reason why the proposed method facilitates to this problem is that the TVFs index is one of the objective functions rather than a stability constraint. The optimal SVC compensation amount can satisfy the demand of the reactive power. So voltage stability problem can be optimized directly. In addition, the rotor stability is satisfied in the optimization as an additional constraint. Then the system can tend to stable well with the optimization result.

In addition, in the dynamic VAR planning optimization, generator rotor angle constraint as (14) needs satisfying. If a power system is unstable under a fault, rotor angle differences between generators may tend to divergent. It means that the generators cannot keep synchronization again. As shown in Fig. 6, rotor angle differences between generators under branch 26-27 fault tend to stable gradually after optimization. A good system needs satisfying transient voltage stability constraint as equation (24). As

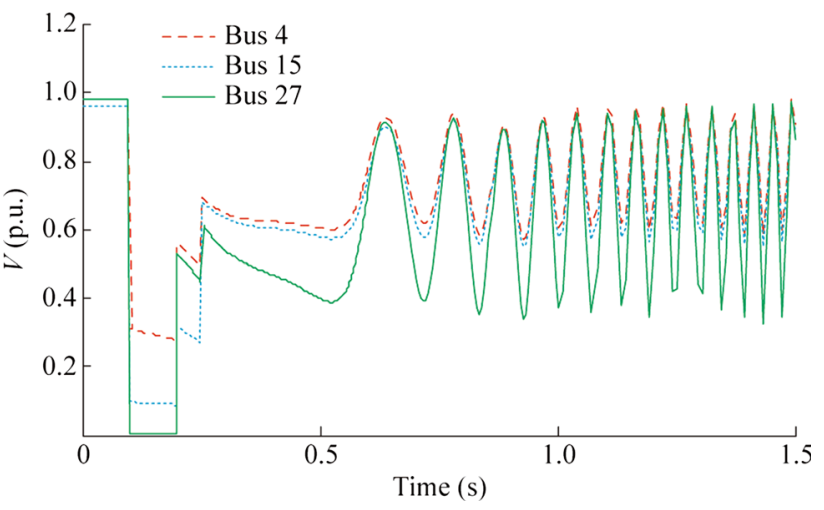

Fig. 4 Transient voltage under branch 26-27 fault before optimization

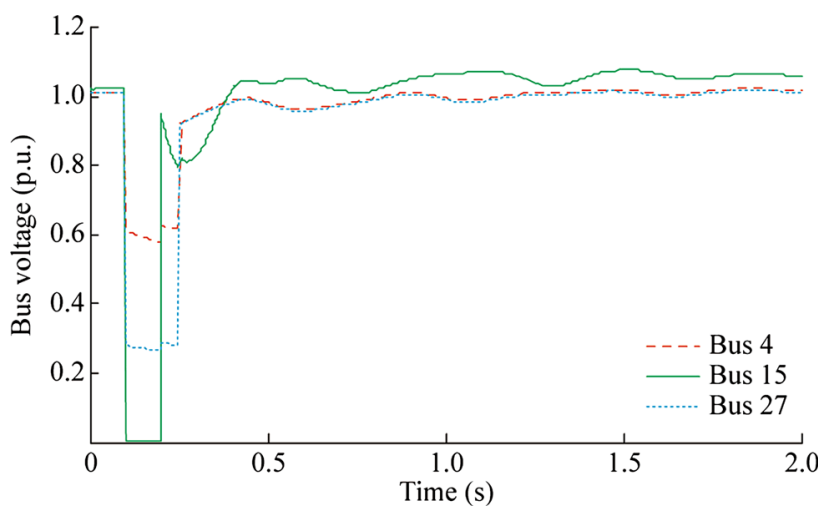

Fig. 5 Transient voltage under branch 26-27 fault after optimization

the result shown in Fig. 6, the transient rotor angles between generators are less than $\pi$. Therefore, the proposed dynamic VAR planning methodology can avoid rotor instability.

Take branch 26-27 fault for an example, the impedance of SVC compensators is shown in Fig. 7. The impedance of SVC3 increases quickly after the fault so that the reactive power is supplemented immediately. When the transient voltage recovers and the system is stable, the impedance of SVC3 recovers to the equilibrium state.

One of the dynamic VAR Pareto optimal solution with SVC is shown in Table 3. The total SVC optimal amount for the three candidate locations is $992 \mathrm{Mvar}$. And the total SVC investment cost is 5.41 million \$/year. The power loss

Table 2 Parameters for dynamic VAR planning optimization

\begin{tabular}{lll}
\hline Symbol & Symbol description & Symbol value \\
\hline$C_{e}$ & Electricity price & $0.099356 \$ / \mathrm{kWh}$ \\
$\zeta$ & Operation hours at the year maximum load & 3600 hours \\
$C_{s v c}$ & Unit capacity investment cost of SVC & 0.05 million $\$ / M v a r$ \\
$\mathrm{~F}_{s v c}$ & Construction investment cost for SVC station & 1.5 million $\$$ \\
$T_{l}$ & Service life of SVC & 10 years \\
\hline
\end{tabular}




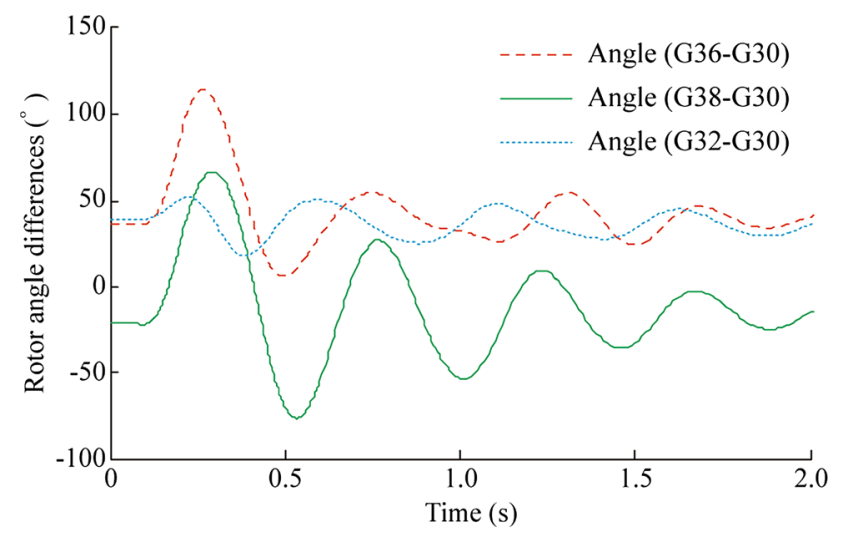

Fig. 6 Generators rotor angle differences under branch 26-27 fault after optimization

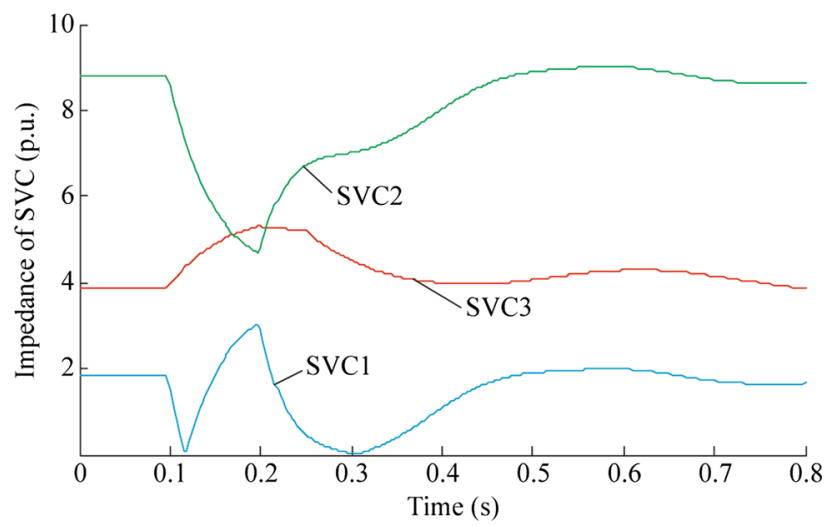

Fig. 7 Reactive power output of the SVC compensators under the optimization solution

cost and SVC investment cost are totally 20.931 million \$/ year. After the dynamic VAR planning optimization, TVFs is 0.0765 , which is decreased by $86.1 \%$ than the initial TVFs 0.5499 before optimization. And the power loss is decreased by $14.2 \%$ than the condition before optimization.

The dynamic VAR optimal solutions under several optimization algorithms (GA, PSO, NSGA II, ITMCO) are shown in Table 4. It can be found that the four algorithms can all make the system tend to stability after faults. The
Table 4 Dynamic VAR optimal solution under several optimization algorithm

\begin{tabular}{llllll}
\hline $\begin{array}{l}\text { Optimization } \\
\text { algorithm }\end{array}$ & \multicolumn{2}{l}{ SVC amount (Mvar) } & $\begin{array}{l}\text { Total cost } \\
\text { (million \$/year) }\end{array}$ & TVFs \\
\cline { 2 - 4 } & SVC1 & SVC2 & SVC3 & & \\
\hline GA & 644 & 171 & 306 & 22.139 & 0.2797 \\
PSO & 651 & 135 & 284 & 21.787 & 0.2812 \\
NSGA II & 626 & 119 & 278 & 21.013 & 0.2764 \\
ITMCO & 582 & 121 & 289 & 20.931 & 0.2723 \\
\hline
\end{tabular}

ITMCO algorithm proposed in this paper with smaller cost is more excellent than other algorithms.

Therefore, the dynamic VAR planning optimization methodology is an effective means to enhance transient voltage stability for failure recovery after faults.

\subsection{A practical power system in China}

For illustrating the implication in the practical power system, a planning power system in China in 2015 shown in Fig. 8 is analyzed in detail. There are 67 buses at $500 \mathrm{kV}, 3$ buses at $1050 \mathrm{kV}$, and 2 HVDC buses at $800 \mathrm{kV}$.

There are 25 branches lined in red in Fig. 8 that will cause voltage instability problem. After the analysis of compensation sensitivity, the best installation locations are bus $3,6,12,16$ and 17 . The compensation sensitivity combined by these candidate locations is 0.0227 , which is the minimum among different combinations of the planning locations. The combination of these candidate locations can enhance the transient voltage stability with small investment amount.

After determined the candidate locations for SVC, the dynamic VAR planning optimization is performed to search the most optimal SVC investment amount. The transient voltage response at bus 3, 6, 7, 17 before and after optimization is illustrated in Fig. 9. It's proved that the transient voltage is stable after installing the optimal SVC compensators. Therefore, failure recovery can be achieved

Table 3 Dynamic VAR optimal solution with SVC for IEEE 39 power system

\begin{tabular}{lll}
\hline Transient voltage stability index & TVFs & 0.0765 \\
SVC amount (Mvar) & SVC1 (bus11) & 582 \\
& SVC2 (bus28) & 121 \\
& SVC3 (bus29) & 289 \\
Operation cost (million \$/year) & Power loss cost & 15.521 \\
Investment cost (million \$/year) & SVC device cost & 4.96 \\
& SVC installation cost & 0.45 \\
Total cost (million \$/year) & Power loss cost and SVC investment cost & 20.931 \\
\hline
\end{tabular}




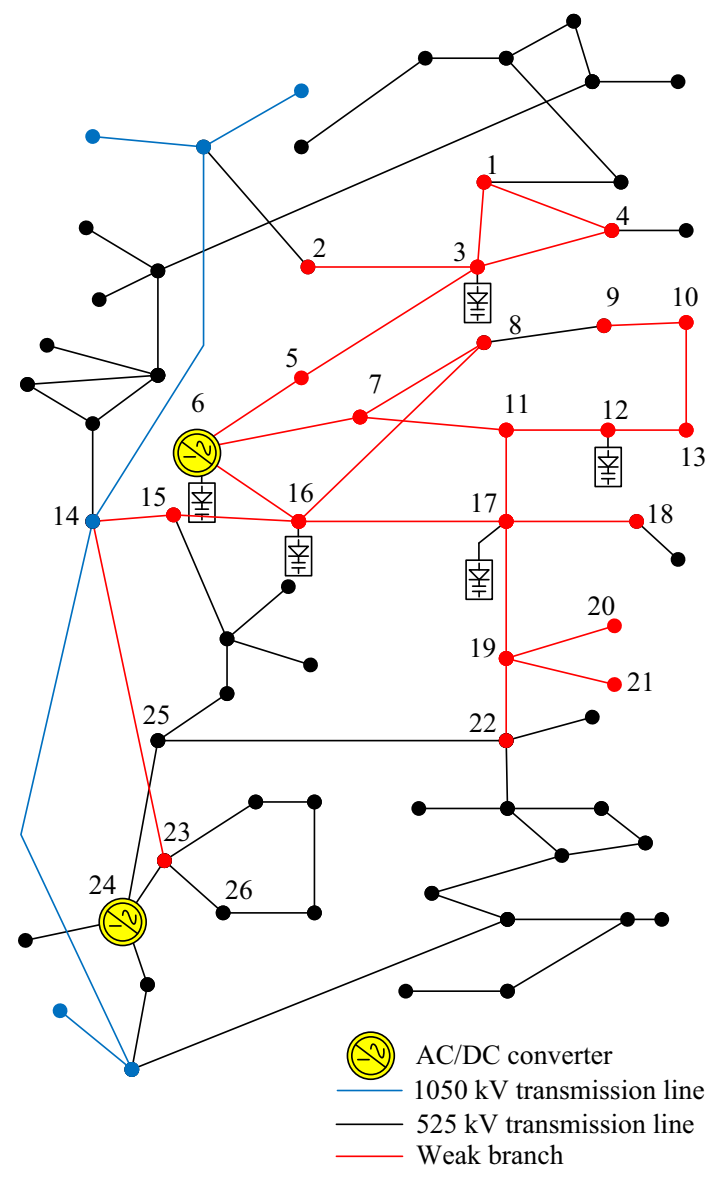

Fig. 8 Topology structure of the practical power system in China

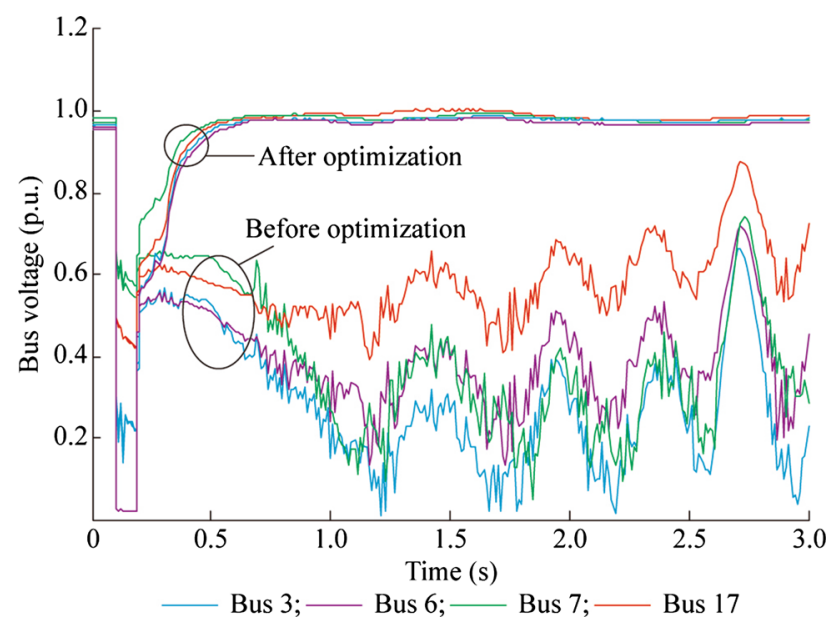

Fig. 9 Transient response of buses $(3,6,7,17)$ before and after optimization

well under the assistance of the planning amount of SVC.

The dynamic VAR optimal solution with SVC for practical power system is shown in Table 5. TVFs after the planning optimization is 0.046. Compared with TVFs
Table 5 Dynamic VAR optimal solution with SVC for practical power system in China

\begin{tabular}{lll}
\hline $\begin{array}{c}\text { Transient voltage } \\
\text { stability index }\end{array}$ & TVFs & 0.046 \\
SVC amount (Mvar) & SVC1 (Bus3) & 878 \\
& SVC2 (Bus6) & 891 \\
& SVC3 (Bus12) & 1167 \\
& SVC4 (Bus16) & 562 \\
& SVC5 (Bus17) & 259 \\
& Power loss cost & 585.8 \\
$\begin{array}{l}\text { Operation cost (million } \\
\text { \$/year) }\end{array}$ & \\
$\begin{array}{l}\text { Investment cost (million } \\
\text { \$/year) }\end{array}$ & SVC device cost & 18.79 \\
Total cost (million \$/ & SVC installation cost & 0.75 \\
year) & Power loss cost and SVC & 605.34 \\
\hline
\end{tabular}

before optimization that is 0.3508 , it's decreased by $86.9 \%$. The total compensation amount is 3757 Mvar. The power loss cost is decreased by $6.081 \%$ after the optimization. And the total cost consisted of power loss and SVC cost is 605.34 million \$/year.

Therefore, the proposed dynamic VAR planning methodology can effectively enhance the transient voltage stability and failure recovery ability.

\section{Conclusion}

A transient voltage assessment index TVF is proposed and a compensation sensitivity approach for dynamic VAR candidate locations is discussed in this paper. Afterwards, a dynamic VAR planning optimization methodology to improve transient voltage stability for failure recovery with the least SVC investment cost and power loss cost is modeled and analyzed based on the improved Tent mapping chaos algorithm. The planning optimization has considered not only transient voltage stability, but also rotor angle stability constraint. Example analysis indicates that the proposed dynamic VAR planning methodology is effective and beneficial for failure recovery after faults.

Acknowledgements This work was supported by the National Basic Research Program (973 Program) of China (No. 2014CB23903) and National Nature Science Foundation of China (No. 51261130473).

Open Access This article is distributed under the terms of the Creative Commons Attribution 4.0 International License (http:// creativecommons.org/licenses/by/4.0/), which permits unrestricted use, distribution, and reproduction in any medium, provided you give appropriate credit to the original author(s) and the source, provide a link to the Creative Commons license, and indicate if changes were made. 


\section{References}

[1] Sode-Yome A, Mithulananthan N, Lee KY (2006) A maximizing loading margin method for static voltage stability in power system. IEEE Trans Power Syst 21(2):799-808

[2] Chang YC (2012) Multi-objective optimal SVC installation for power system loading margin improvement. IEEE Trans Power Syst 27(2):984-992

[3] Ghahremani E, Kamwa I (2013) Optimal placement of multipletype FACTS devices to maximize power system loadability using a generic graphical user interface. IEEE Trans Power Syst 28(2):764-778

[4] Ray B (2003) FACTS technology application to retire aging transmission assets and address voltage stability reliability challenges in San Francisco bay area. In: Proceedings of 2003 IEEE power energy society transmission and distribution conference and exposition, Dallas, USA, 7-12 September 2003, pp 1412-1419

[5] Ray B (2006) Recent experience at PG\&E with FACTS technology application. In: Proceedings of 2006 IEEE power engineering society transmission and distribution conference exhibition, Dallas, USA, 21-24 May 2006, pp 1113-1120

[6] Liu HF, Krishnan V, McCalley JD et al (2014) Optimal planning of static and dynamic reactive power resources. IET Gener Transm Distrib 8(12):1916-1927

[7] Xu Y, Dong ZY, Meng K et al (2014) Multi-objective dynamic VAR planning against short-term voltage instability using a decomposition-based evolutionary algorithm. IEEE Trans Power Syst 29(6):2813-2822

[8] Krishnan V, Liu HF, McCalley JD (2009) Coordinated reactive power planning against power system voltage instability. In: Proceedings of IEEE PES power systems conference exposition, Settle, USA, 15-18 March 2009, pp 1-4

[9] Sapkota B, Vittal V (2010) Dynamic Var planning in a large power system using trajectory sensitivities. IEEE Trans Power Syst 25(1):461-469

[10] Paramasivam M, Salloum A, Ajarapu V et al (2013) Dynamic optimization based reactive power planning to mitigate slow voltage recovery and short term voltage instability. IEEE Trans Power Syst 28(4):3865-3873

[11] Tiwari A, Ajjarapu V (2011) Optimal allocation of dynamic VAR support using mixed integer dynamic optimization. IEEE Trans Power Syst 26(1):305-314

[12] Alejandro PM, Fuerte-Esquivel CR, Daniel RV (2010) Global transient stability-constrained optimal power flow using an OMIB reference trajectory. IEEE Trans Power Syst 25(1):392-403

[13] Rafael ZM, Cutsem TV, Milano F et al (2010) Securing transient stability using time-domain simulations within an optimal power flow. IEEE Trans Power Syst 25(1):243-253

[14] Xu Y, Dong ZY, Meng K et al (2012) A hybrid method for transient stability-constrained optimal power flow computation. IEEE Trans Power Syst 27(4):1769-1777

[15] Ye CJ, Huang MX (2015) Multi-objective optimal power flow considering transient stability based on parallel NSGA-II. IEEE Trans Power Syst 30(2):857-866

[16] Alejandro PM, Fuerte-Esquivel CR, Daniel RV (2011) A new practical approach to transient stability-constrained optimal power flow. IEEE Trans Power Syst 26(3):1686-1696

[17] Rosehart W, Cañizares CA, Quintana VH (2002) Effect of detailed power system models in traditional and voltage stability constrained optimal power-flow problems. IEEE Power Eng Rev 22(12):59
[18] Valipour M (2016) Optimization of neural networks for precipitation analysis in a humid region to detect drought and wet year alarms. Meteorol Appl 23(1):91-100

[19] Yannopoulos SI, Lyberatos G, Theodossiou N (2015) Evolution of water lifting devices (pumps) over the centuries worldwide. Water 7:5031-5060

[20] Valipour M, Sefidkouhi MAG, Eslamian S (2015) Surface irrigation simulation models: a review. Int J Hydrol Sci Technol 5(1):51-52

[21] Valipour M (2012) Sprinkle and trickle irrigation system design using tapered pipes for pressure loss adjusting. J Agric Sci 4(12):125-133

[22] Khasraghi MM, Sefidkouhi MAG, Valipour M (2015) Simulation of open- and closed-end border irrigation systems using SIRMOD. Arch Agron Soil Sci 61(7):929-941

[23] Valipour M (2012) Comparison of surface irrigation simulation models: full hydrodynamic, zero inertia, kinematic wave. J Agric Sci 4(12):68-74

[24] Wang RQ, Zhang CH, Li K (2011) Multi-objective genetic algorithm based on improved chaotic optimization. Control Decis 26(9):1391-1397

Di YANG received the B.S. degree in electrical engineering from Shijiazhuang Railway Collage, Shijiazhuang, China, in 2006, and the M.S. degree in electrical engineering from Northwest Polytechnic University, Xi'an, China, in 2009, and the Ph.D. degree from Shanghai Jiao Tong University, Shanghai, China, in 2016, respectively. He is currently working at State Grid Pudong Power Supply Company, SMEPC, Shanghai, China. His main research interests include voltage stability analysis and reactive power planning optimization.

Haozhong CHENG received the B.S., M.S., and Ph.D. degrees in power systems from Shanghai Jiao Tong University, Shanghai, China, in 1983, 1985, and 1998, respectively. He was a former Visiting Professor with Iowa State University, Ames, IA, USA, and the University of Missouri-Rolla, Rolla, MO, USA. He is working as a professor in the Department of Electrical Engineering, Shanghai Jiao Tong University, Shanghai, China. His research interests include mainly power system planning, voltage stability, harmonics, operation.

Zeliang MA received the B.S. degrees in power systems from Shanghai Jiao Tong University, Shanghai, China, in 1983. He is working in East China Power Grid Corp, Shanghai, China. His research interests include power grid planning and management.

Liangzhong YAO received the M.S., and Ph.D. degrees in power system engineering in the Department of electrical engineering of Tsinghua University, Beijing, China, in 1989 and 1993. He is working in China Electric Power Research Institute, Beijing, China. His research interests include power system analysis and large scale wind farm integration technology.

Zhonglie ZHU received the M.S. degrees in power systems from Shanghai Jiao Tong University, Shanghai, China, in 1987. He is working in East China Power Grid Corp, Shanghai, China. His research interests include power grid planning and management. 